\title{
Nanoscale-Technologies as Subjects of Responsible Research and Innovation
}

\author{
Stephan Lingner • John Weckert
}

Received: 6 June 2016 / Accepted: 9 June 2016 / Published online: 30 June 2016

C) Springer Science+Business Media Dordrecht 2016

The term "Responsible Research and Innovation" (RRI) has been central in European research and innovation policy in the current decade although less so in other countries, for example in the USA and Australia. It comprises aims such as established methods of technology reflection, for example technology assessment and practical ethics, among others. However, RRI claims a specificity with respect to dealing with the inevitably disruptive potential of "creative destruction," which is the essence of innovation in the Schumpeterian sense. The obvious tension between competitive vs. social values of innovation as well as its unwanted or uncertain side-effects gives reason for corresponding responsibility deliberations. Related societal risks and uncertain outcomes of innovation are therefore subjects of attempts to assess and balance opportunities and risks of realizing new options with emerging technologies. Additionally, there are just expectations of the taxpayers for relevant as well as acceptable research and innovation that has been publicly funded; corresponding benefits should thus be in the interest of society. Moreover, responsible conduct of innovation can be also prudent for the actors because public acceptance of new technologies can be seen as a prerequisite for marketable

S. Lingner $(\bowtie)$

EA European Academy of Technology and Innovation

Assessment, Bad Neuenahr-Ahrweiler, Germany

e-mail: stephan.lingner@ea-aw.de

J. Weckert

Charles Sturt University, Canberra, Australia products and services and thus for economic benefits and amortization of investments. In such cases, the impetuses of the sellers and the demand-side might even convergence to some extent.

Nevertheless, the notion of responsibility - although implicitly and supposedly clear-seems underdetermined with respect to the objective to give guidance to the "responsible" actors in research and innovation. The main reason underlying this is that any overarching concept of responsibility relies on an underlying plurality of presuppositions, preferences, and values and thus by related epistemic and normative conflicts. In order to clarify the concept, some authors have attempted to specify the dimensions of responsibility in more detail [1-3]. However, some residual vagueness of the concept remains, for instance in case of von Schomberg's attempts to specify responsible innovation by desirability criteria like "positive outcomes," which are themselves debatable. Currently, the concept of responsibility seems to be mainly challenged (a) by the attribution problem of individual responsibility in collective innovation actions, (b) by the profound uncertainty of the consequences of acting or non-acting in a complex and dynamic world, and (c) by normative plurality and ambivalence [4]. Responsible innovation is therefore less of a moral black-box approach than of laborious societal evaluation and negotiation processes of case-specific research and innovation potentials.

The subjects of RRI are many in the context of new and emerging technologies. The case of nanoscale technologies is paradigmatic here as they raise not only hopes or even hypes but also fears, which are reflected by 
related utopian views on one hand and by dystopian narratives on the other hand. The distant future perspectives on these technologies are confusing because of their abstract and uncertain realizations on nano-levels which are remote from daily life experience and individual control, thus allowing for speculation in either direction. This profound uncertainty on "nano-futures" challenges the ethical, social, and environmental assessment of converging nanotechnologies and thus any attempt to balance their benefits and risks for society. This specific challenge has also given a reason for dedicated RRI case studies on nanotechnology and synthetic biology within the project "Promoting Global Responsible Research and Social and Scientific Innovation" [5] funded by the European Commission. This project established a worldwide RRI network while serving as a fact-finding mission on global views and convergence potentials of responsible research and innovation policies. The results have also led to academic contributions on case-specific best practices of RRI, which are published in this present special section of NanoEthics. The following articles reflect the scientific perspectives on responsibility in research and innovation in Europe and abroad from the international partners of the project. The first focuses on RRI itself and uses an aspect of nanotechnology as its main example. The next two are in the bio-domain and concerned primarily with synthetic biology and the final one returns to RRI more generally.

In the first paper Responsible, Inclusive Innovation and the Nano-divide, Doris Schroeder (UK) et al. argue that RRI and justice can both provide similar ethical conclusions on the nano-divide problem but that RRI is a useful supplement to the justice argument. It (RRI) can be seen as a mediator concept between competitive and justice considerations. Systems of innovation also play an important role in RRI here by providing empirical evidence regarding its claims. The authors start with the clarification of key terms as used within the RRI debate, followed by a critical analysis of the relevant concepts of RRI, inclusive innovation, and innovation systems with respect to their specific potential for handling the nanodivide. Societal needs and desirability for innovation as well as its responsive arrangement are central here. It should be noted that Schroeder et al. define societal desirability in global terms; it relates to all people everywhere ${ }^{1}$. Finally, they conclude that both concepts of RRI

\footnotetext{
${ }^{1}$ In the final paper of the special section, Weckert et al. use it as referring only to a particular society.
}

and inclusive innovation are capable of adding the necessary moral values into the appropriate governance of innovation systems. Traditional ethical justice concepts could be considered as well but the case of the nanodivide shows that RRI addresses the normative questions of broad access towards desirable nano-technologies much better than mere justice deliberations alone. RRI serves here as a mediator concept between societal and economical value systems. Moreover, the economic potential of the European RRI approach might be further enhanced by the descriptive concept of innovation systems. The authors stress that the idea of inclusive innovation could bridge the gap here between descriptive and normative concepts of innovation

The article of Sarah Laird (USA) and Rachel Wynberg (South Africa) explores the topical relation of Responsible Research and Innovation and the Convention on Biological Diversity (CBD) with the aim to extract further Lessons for Emerging Technologies from this linkage in the field of synthetic biology. The authors show that on one hand v. Schomberg's RRI values are implicitly also part of the CBD; that holds especially true for the more recent measures of the follow-up "Nagoya Protocol" and related ongoing negotiation processes on "Access and Benefit Sharing (ABS)." On the other hand, the emerging field of synthetic biologyalso a subject of the Nagoya Protocol-clearly addresses the normative anchor points of RRI in so far as it promises new desirable products and processes while letting questions on possible environmental or health risks still open. Similarly, ethical concerns for the concept of life and for the human condition remain largely unanswered so far with respect to this new strand of bioengineering. Moreover, weighing and balancing the overall utility of synthetic biology for the society is a challenging task when considering the fast advance of research and development in that field. It is especially a problem for regulation to keep up with this dynamic development, especially with regard to the limited competences, capacities, and resources of regulatory bodies as well as with regard to the solution of juridical conflicts thereupon. In this context, Laird and Wynberg recognize additional weaknesses of the previous ABS processes as the latter ignored the conceptual diversity and scope of synthetic biology, related systemic complexities, and geopolitical implications. The authors complain also about the ineffectiveness of the ABS regulations from their formal structure and downstream processes. However, the ABS also enabled more 
flexible dialogues and reflections between the different stakeholders on the conduct of responsible research and development within the domain of the CBD. The authors argue therefore for a continuation and empowerment of corresponding discussions and deliberations on national and global levels about the potential benefits and harms of synthetic biology as well as on related uncertainties and ethical constraints. RRI would thus enter into practice while enforcing reflective, transparent, and anticipative processes with respect to the impacts of an emerging technology.

The next contribution, Science Policy and Concomitant Research in Synthetic Biology - Some Critical Thoughts by Kristin Hagen (Germany) is also in the bio-domain. Her starting point is the observation that this new technology field has so far generated surprisingly little public controversy. The author identifies several strategies employed by the actors in that field ranging (a) from claiming the burden of scientific proof on any critical argument (b) over reference to already existing regulations on genetic engineering (while denying new challenges from synthetic biology) (c) to implicit masking of specific topics of concern by initiatives of self-governance and engagement with other disciplines. The often hidden heterogeneity of the concept of synthetic biology and its societal implications seem to be a challenge for the notion of responsible research and innovation as well as to established reflective frameworks like technology assessment. However, synthetic biology and its interdisciplinary "tribes" of engagement have already become part of the "technoscientific promise-culture" while targeting the grand challenges of our society as well as the bio-economic agendas of our time. Hagen notices some sort of ironic co-existence of 'opening up and closing down' of related debates, maxims and responsibilities by embedding them upstream in the (pretended) RRI process of synthetic biology. She questions whether RRI in this form might compromise independent investigations through growth-led innovation and premature policy values. The author concludes with a plea for better governance and enough space for open debate of controversial views in science and politics of synthetic biology. Corresponding RRI processes should consider safety, security, and legitimacy issues in the light of pending uncertainties and ethical implications of that emerging technology. Finally, she argues that public-funded research should not be guided by vested interests - at least in the realm of responsibility.
The final paper by John Weckert et al. (Australia) acknowledges the social value and utility of responsible research and innovation in-line with the "inventors" of the concept of RRI. Nevertheless, the authors draw attention to A problem with social desirability as a component of Responsible Research and Innovation as an integral part of the specific RRI definition from von Schomberg [3]. The authors argue that the desirability criterion is ambiguous particularly with respect to its scope $^{2}$. Moreover, they see internal goal conflicts with the ethical acceptability criteria, which are also put forward by von Schomberg and other RRI proponents. Weckert and co-authors develop their argument along the value chain of scientific research, technological development, and societal innovation and its problem solving potential. For instance, tailored engineering of bacteria might be desirable as future means to clean-up contaminated sites. But their ecological behavior is unknown and their uncontrolled spreading might endanger the larger environment or even the society. The question is, if there were other, less risky alternatives to cope with severe contaminations. Reflective equilibria of the consequences could lead to legitimate decisions of either strategy. Nevertheless, "desirable" strategies might also prioritize those solutions which are in the national interest while implicitly affecting other parties. This is the case when the commonly used argument "If we don't do it, others will" is put forward as a justification, albeit, a dubious one. The authors demonstrate by this example the tension between the desirability criterion and von Schomberg's other normative RRI "anchor points." Therefore, setting up new research agendas and innovation policies together with considerations of societal desirability has to be done context-sensitively while caring for compliance with fundamental ethical principles as long as responsibility will be aimed at as the overarching goal. The "If we don't others will" argument might be dubious but is commonly used and is seen as powerful by those employing it so should not be overlooked, or so the authors maintain.

\section{Concluding Remarks}

Responsible Research and Innovation-although a necessity in the domain of emerging technologies - needs

\footnotetext{
${ }^{2} \mathrm{Cf}$ the discussion of societal desirability of Schroeder et al. in the first paper of the special section.
} 
further refinement with regard to conceptual issues and practical embeddedness in order to become what it aims at - a game-changer. The contributions to this special section might pave the way forward: a challenge for the future will be for careful specification of the desirability criterion of the RRI concept as far as von Schomberg's notion will be followed in public research and innovation policy (see also critical statements of [6]). Another crucial and still controversial point-even among the authors of this special section - is the question of whether the inclusiveness prerogative in RRI should be fostered while enabling effective co-design of new technologies or whether this will lead to complicity and to quieting of critical debates thereupon (see also [7] on this issue). These and similar needs for clarification of the quite ambitious RRI concept have to be dealt with before the concept can be expected to enter the arena of practical technology governance.

Acknowledgement This contribution was written with support from the European Community's Seventh Framework Programme under grant number 321400 .

\section{References}

1. Grunwald A (2011) Responsible innovation: bringing together technology assessment, applied ethics, and STS research. Enterprise and Work Innovation Studies 7:9-31

2. Stilgoe J, Owen R, MacNaghten P (2013) Developing a framework for responsible innovation. Research Policy 42(9):15681580

3. Von Schomberg R (2013) A vision of responsible innovation. In: Owen R, Bessant JR, Heintz M (eds) Responsible innovation: managing the responsible emergence of science and innovation in society. Wiley, Chichester, pp 51-74

4. Lingner S (2015) Exploring 'responsibility' in research and innovation. In: Bowman DM, Dijkstra A, Fautz C, Guivant J, Konrad K, Van Lente H, Woll S (eds) Practices of innovation and responsibility: insights from methods, governance and action. IOS Press, Amsterdam, pp 99-110

5. PROGRESS (2016) Promoting global responsible research and social and scientific innovation. Coordination and Support Action of the European Commission (grant no. 321400). http://www.progressproject.eu Accessed 2 June 2016

6. Coles D, Engelhard M, Han B, Kumar A, Laas K, Ladikas M, Lin R, Schroeder D, Srinivas R, van Niekerk J, Walker M, Weckert J (2014) RRI country requirements matrix Nanotechnology, synthetic biology, ICT. Report for FP7 Project "PROGRESS". http://www.progressproject.eu Accessed 2 June 2016

7. Zwart H, Landeweerd L, van Rooij A (2014) Adapt or perish? Assessing the recent shift in the European research funding arena from 'ELSA' to 'RRI'. Life Sciences, Society and Policy 10:1-19 